\title{
RELATO DE CASO
}

\section{SISTEMA NERVOSO AUTÔNOMO INTRACARDÍACO EM CASO HUMANO FATAL DE DOENÇA DE CHAGAS AGUDA}

\author{
Rogério de Almeida-Ribeiro, Delmar Muniz Lourenço Junior, João Carlos \\ Pinto Dias, Maria Aparecida Shikanai-Yasuda, Edmundo Chapadeiro e \\ Edison Reis Lopes
}

\begin{abstract}
Procedeu-se à análise do sistema nervoso autônomo intracardíaco (SNAIC) em 150 cortes histológicos obtidos a partir de fragmentos atriais de homem de 74 anos, falecido de insuficiência cardíaca consecutiva à doença de Chag łs aguda (DCA), provavelmente adquirida por via digestiva. Em 10 lâminas havia discretos infiltrados de mononucleares em torno de gânglios elou filetes nervosos sem alterações morfológicas significativas dos neurônios; em um preparado constatou-se ganglionite e periganglionite, de moderada intensidade, associadas a alterações neuronais. Epicardite focal, em geral discreta, foi observada em todas as lâminas. Os achados sugerem que a inflamação dos gânglios e fibras do SNAIC na DCA ocorre, pelo menos em parte, da propagação da epicardite adjacente e, que mesmo nos casos fatais da tripanosomose cruzi as lesões morfológicas do SNAIC podem ser discretas.
\end{abstract}

Palavras-chaves: Doença de Chagas. Cardiopatia chagásica. Sistema nervoso autônomo. Sistema nervoso autônomo intracardíaco. Tripanosomose cruzi.

A análise morfológica, qualitativa e quantitativa, do sistema nervoso autônomo intracardíaco (SNAIC) na fase aguda da doença de Chagas (DC) humana foi realizada apenas em três $\operatorname{casos}^{269}$. Em todos havia lesões dos gânglios e filetes nervosos acompanhados de intensa destruição neuronal.

Tendo necropsiado chagásico falecido na fase aguda da DC - adquirida provavelmente por via oral - e constatado raras e discretas lesões do SNAIC pareceu-nos de interesse relatar os achados pelas suas implicações de ordem patogenética, fisiopatológica e epidemiológica.

\section{RELATO DO CASO}

Homem de 74 anos, natural da Paraíba, hipertenso, com quadro clínico e laboratorial de doença de Chagas aguda (DCA) a qual levou à

\footnotetext{
Curso de Pós-Graduação em Patologia Humana da Faculdade de Medicina do Triângulo Mineiro, Departamento de Doenças Infecciosas e Parasitárias da Faculdade de Medicina da Universidade de São Paulo e Divisão de Doença de Chagas, Ministério da Saúde, Brasilia.

Endereço para correspondência: Prof. Edison Reis Lopes, Pós-Graduação em Patologia Humana, Faculdade de Medicina do Triângulo Mineiro, Pça Thomaz Ulhoa 706, 38025-050 Uberaba, MG, Brasil.

Recebido para publicação em 20/11/92.
}

insuficiência cardíaca e ao óbito. Este caso associado a outros 25 , com infecção chagásica possivelmente adquirida por via oral, foi motivo de publicação anterior ${ }^{10}$. Dentre os achados ressalta-se que o eletrocardiograma revelou bloqueio da divisão ânterosuperior e distúrbios de ramo direito.

A necrópsia parcial mostrou coração aumentado de volume, especialmente às custas do ventrículo esquerdo. O epicárdio, em geral liso e brilhante, apresentava nas superfícies átrio-ventriculares, placas e faixas lineares, esbranquiçadas ao longo de alguns ramos coronários. As cavidades cardíacas eram dilatadas e o miocárdio vermelho-escuro, homogêneo e brilhante; o endocárdio parietal era liso e brilhante, exceto em correspondência com a região vorticilar esquerda, onde havia trombo parietal recente. As válvulas eram morfologicamente normais.

Microscopicamente, o miocárdio mostrava inflamação com as características da miocardite chagásica aguda $a^{3}$, com abundantes formas amastigotas do Trypanosoma cruzi, associada à fibrose miocárdica isquêmica (doença cardíaca crônica isquêmica). No endocárdio parietal havia pequenos focos de infiltrado de mononucleares e trombose recente na região vorticilar. O epicárdio, em grande parte de sua extensão, apresentava-se de aspecto normal (Figura 1). Em algumas áreas havia múltiplos e pequenos focos de epicardite exsudativa (Figura 2) associados 
Relato de Caso. Almeida-Ribeiro $R$, Lourenço Junior DM, Dias JCP, Shikanai-Yasuda MA, Chapadeiro E, Lopes ER. Sistema nervoso autônomo intracurdiaco em caso humano fatal de doença de Chagas aguda. Revista da Sociedade Brasileira de Medicina Tropical 26:35-38, jan-mar, 1993.

ou não a áreas de fibrose decorrente de antiga epicardite (placas e faixas).

A análise do SNAIC foi realizada em 135 cortes histológicos colhidos e corados dentre 945 , retirados de anel em torno da veia cava superior, conforme metodologia por nós já empregada ${ }^{5}$. Nos 135 cortes histológicos o epicárdio mostrava, também, discretos focos de infiltrado de mononucleares os quais, raras vezes, se situavam em torno de gânglios e filetes nervosos (periganglionite e perineurite; Figura 3). A maioria dos gânglios, entretanto, não apresentava alteraçōes (Figura 4). Nos 135 cortes histológicos contaram-se nos vários gânglios 418 neurônios os quais, raramente, exibiam discretas lesões morfológicas como hipercromasia e vacuolização. Examinou-se ainda o SNAIC em 15 outras áreas atriais, das quais retirou-se um corte histológico de cada. As alterações constatadas eram similares às anteriormente descritas, exceto em uma onde a epicardite focal envolvia e invadia gânglio (periganglionite e ganglionite; Figura 5). Neste gânglio, ao contrário dos demais, havia alterações regressivas neuronais mais acentuadas (tumefação, retração do citoplasma) e, aparentemente, destruição de células ganglionares (Figura 6).

À necrópsia constatou-se ainda: 1. miosite focal, com presença de amastigotas do $T$. cruzi nas porçōes proximais do tubo digestivo; 2 . órgãos cardíacos; 3. arteriosclerose moderada da aorta, seus grandes ramos e coronárias; 4 . nefrosclerose arteriolar benigna.

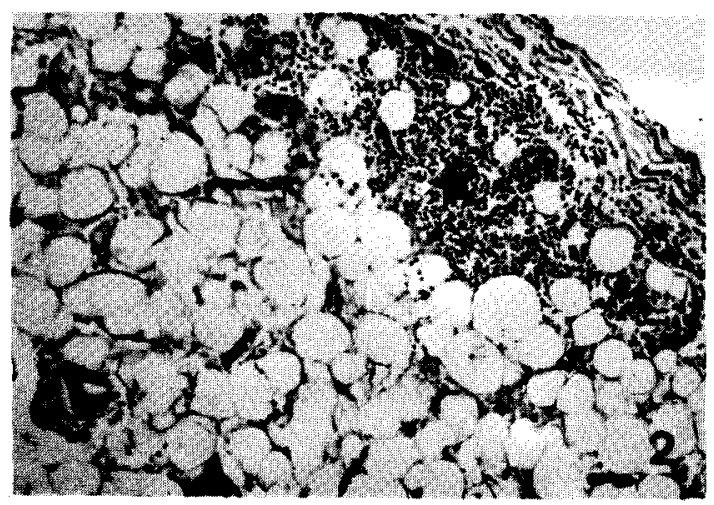

Figura 2 - Foco de exsudato inflamatório (asterisco) em epicárdio atrial. Hematoxilina-eosina 100x.

do epicárdio, de gânglio (seta) e de filete nervoso (cabeça de seta). Hematoxilinaeosina $40 x$.

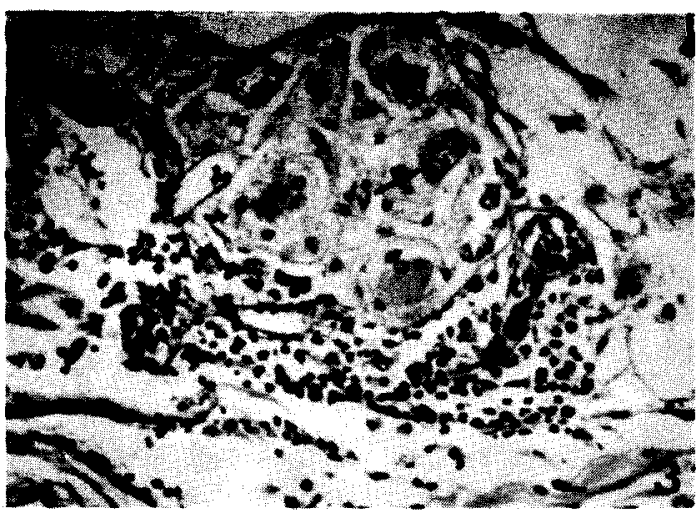

Figura 3 - Gânglio subepicárdico. Notar a periganglionite e a integridade dos neurônios (seta). Hematoxilina-eosina $400 x$.

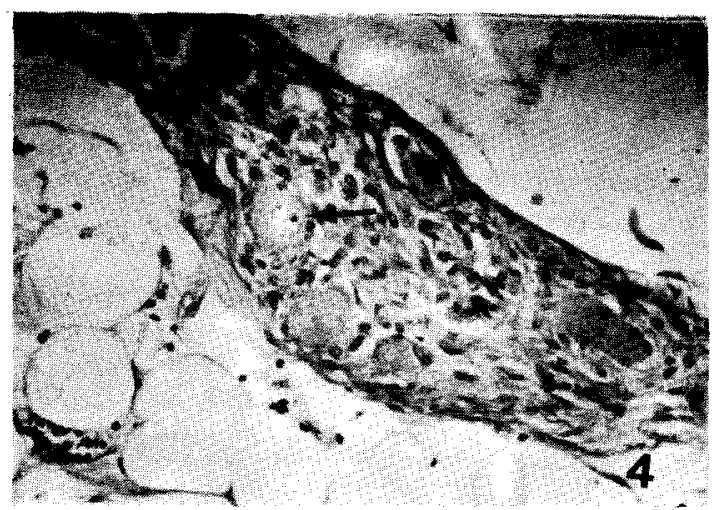

Figura 4-Gânglio subepicárdico sem alterações microscópicas. Aseta indica neurônioíntegro. Hematoxilina-eosina $400 x$. 


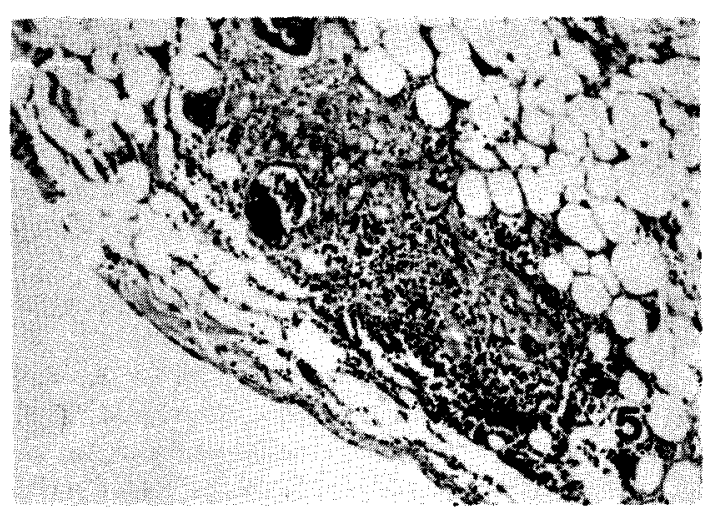

Figura 5 - Corte do átrio direito mostrando epicardite que se estende a gânglio com periganglionite e ganglionite intensas. As setas indicam neurônios, um dos quais alterado. Hematoxilina-eosina $100 x$.

\section{DISCUSSÃO}

Os achados do presente caso mostram que as lesões do SNAIC na DCA humana podem ser raras e discretas, ao contrário do relatado nos casos humanos anteriormente estudados ${ }^{269} \mathrm{e}$ em alguns modelos experimentais ${ }^{111}$, onde a epicardite $e$ as lesões dos gânglios e dos filetes nervosos eram freqüentes e graves.

Alguns dos achados do caso ora relatado podem contribuir para o melhor conhecimento da patogênese e fisiopatologia da cardiopatia chagásica, como por exemplo o papel que nela desempenham as lesões do SNAIC. Infelizmente, por ter sido destruída parte do tecido localizado em torno da veia cava superior, não pudemos realizar a análise quantitativa completa dos neurônios do SNAIC, o que permitiria comparar os resultados do presente caso com os demais da literatura. Entretanto, a análise feita nos parece suficiente para indicar que as lesões dos gânglios e nervos cardíacos eram raras, em geral discretas e a destruição neuronal mínima. Isto pode explicar, pelo menos em parte, os achados divergentes entre autores que estudaram a denervação cardíaca na $\mathrm{DC}^{5}$ 7. Também somos levados a especular sobre o significado funcional da intensa inflamação com denervação de um único gânglio, dentre os vários examinados. James e cols ${ }^{4}$, em duas mulheres não chagásicas, falecidas súbita e inesperadamente, encontraram ganglionite, predominantemente na região do nodo sinusal e, segundo os mesmos

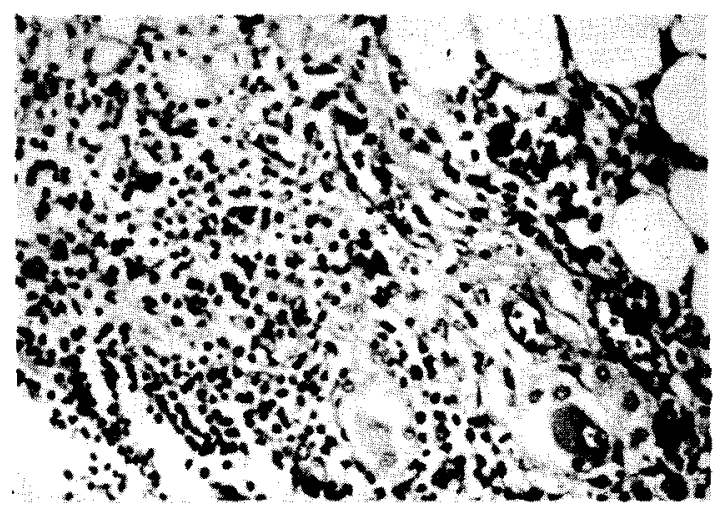

Figura 6 - Detalhe da Figura 5. Hematoxilina-eosina $400 x$.

autores, o significado funcional do achado não pôde ser esclarecido, mas por sua localização poderia causar instabilidade elétrica fatal. Infelizmente no caso ora em discussão não pudemos estabelecer a localização exata do gânglio com inflamação intensa e denervação. O único dado topográfico que temos indica que se localizava no átrio direito em área não em torno da veia cava superior. Em nosso entender, entretanto, mesmo que se soubesse a localização exata do gânglio não se poderiam tirar maiores conclusões sobre o papel das lesões que apresentava, visto que os conhecimentos morfofuncionais atuais sobre o SNAIC não permitem determinar as conseqüências que as lesões localizadas ou sistematizadas dos gânglios cardíacos produzem na cardiopatia chagásica.

$O$ presente relato mostra, ainda, que no único gânglio em que se observou inflamação grave com destruição neuronal, havia relação de continuidade entre a epicardite e a ganglionite. Isto confirma observação anterior de que o processo inflamatório das estruturas do SNAIC ocorre, pelo menos em parte, por extensão da epicardite vizinha.

As diferenças morfológicas observadas no caso ora relatado e naqueles descritos por Becker ${ }^{2}$, Lopes e $\operatorname{cols}^{6}$ e Miziara e cols ${ }^{9}$ poderiam ser atribuídas às diferentes vias de inoculação do $T$. cruzi: oral, ne presente caso, transfusional no de Becker e natural nos demais. Finalmente, pode-se também especular se o zimodema do $T$. cruzi seria o mesmo nos quatro casos, visto que alguns estudos sugerem que $T$. cruzi com diferentes zimodemas podem ser responsáveis por diferentes formas anatomoclínicas da $\mathrm{DC}^{8}$. 
Relato de Caso. Almeida-Ribeiro R, Lourenço Junior DM, Dias JCP, Shikanai-Yasuda MA, Chapadeiro E, Lopes ER. Sistema nervoso autônomo intracardiaco em caso humano fatal de doença de Chagas aguda. Revista da Sociedade Brasileira de Medicina Tropical 26:35-38, jan-mar, 1993.

\section{SUMMARY}

An analysis of the intracardiac autonomic nervous system (ICANS) has been made in 150 histological sections obtained from atrial fragments of a 74 year-old man who died of cardiac failure, as a consequence of acute Chagas' disease (ACD), probably acquired via digestive tract. Small quantities of mononuclear infiltrate around ganglia and/or nerve branches without significant morphologic alterations of the neurons were found in 10 slides; another slide showed ganglionitis and periganglionitis of moderate intensity associated to neuronal alterations. Focal epicarditis, usually of slight degree, was observed in all slides. The findings suggest: a) that the inflammation of ganglia and fibers of the ICANS in the ACD occurs at least in part by expansion from adjacent epicarditis; $b$ ) that even in the fatal cases of the tripanosomiasis cruzi the pathologic lesions of the ICANS may be slight.

Key-words: Chagas'disease. Chagasic cardiopathy. Autonomic nervous system. Intracardiac autonomic nervous system. Trypanosomiasis cruzi.

\section{REFERENCIAS BIBLIOGRÁFICAS}

1. Andrade ZA, Andrade SG. Patologia. In: Brener Z, Andrade ZA (ed) Trypanosoma cruzi e Doença de Chagas. Guanabara Koogan, Rio de Janeiro p.199248, 1979.

2. Becker PFL. Moléstia de Chagas aguda acidental (por transfusão de sangue de doador chagásico). Revista do Instituto de Medicina Tropical de São Paulo 17:187-198, 1975.

3. Chapadeiro E, Tafuri WL, Lopes ER. Anatomia patológica da cardiopatia aguda. In: Cançado JR, Chuster M (ed) Cardiopatia chagásica. Fundação Carlos Chagas, Belo Horizonte p.29-32, 1985.

4. James TN, Zipes DP, Finegan RE, Eisele JN, Carter
JE. Cardiac ganglionitis associated with sudden unexpected death. Annals of 'Internal Medicine 91:727-730, 1979.

5. Lopes ER. Contribuição ao estudo dos gânglios cardíacos (sistema nervoso autônomo) em chagásicos crônicos. Tese de Doutorado, Faculdade de Medicina do Triângulo Mineiro, Uberaba, Minas Gerais, 1965.

6. Lopes ER. Tafuri WL, Bogliolo L, Almeida HO, Chapadeiro E, Raso P. Miocardite chagásica aguda humana (ganglionite subepicárdica; agressão a fibra cardíaca por linfócitos; relação entre amastigotas e fibra muscular). Revista do Instituto de Medicina Tropical de São Paulo 19:283-360, 1977.

7. Lopes ER, TafuriWL. Involvement of the autonomic nervous system in Chagas heart disease. Revista da Sociedade Brasileira de Medicina Tropical 16:206$212,1983$.

8. Miles MA, Cedillos RA, Povoa MM, De Souza AA, Prata A, Macedo V. Do radically dissimilar Trypanosoma cruzi strains (zymodemes) cause Venezuelan and Brazilian forms of Chagas' disease? Lancet 1:1338-1340, 1981.

9. Miziara HL, Santos BG, Lopes ER, Tafuri WL, Chapadeiro E. Contribuição ao conhecimento do quadro anatomopatológico do coração na doença de Chagas. Revista da Sociedade Brasileira de Medicina Tropical 17:101-105, 1984.

10. Shikanai-Yasuda MA, Marcondes CB, Guedes LA, Siqueira GS, Barone AA, Dias JCP, Amato Neto V, Tolezano JE, Peres BA, Arruda Jr ER, Lopes MH, Shiroma M, Chapadeiro E. Possible oral transmission of acute Chagas' disease in Brazil. Revista do Instituto de Medicina Tropical de São Paulo 33:351357, 1991.

11. Tafuri WL. Light and electron microscope studies of the autonomic nervous system in experimental and human. American trypanosomiasis. Virchows Archives für Pathologische Anatomie (Berlin) 354:316-319, 1971. 